\title{
REDUCTIVE WEAK DECOMPOSABLE OPERATORS ARE SPECTRAL
}

\author{
KOTTARÓ TANAHASHI
}

\begin{abstract}
We show that if a bounded linear operator $T$ on a complex Hilbert space is reductive and weak decomposable, then $T$ is a spectral operator with a normal scalar part. This is a generalization of a result due to Jafarian [3].
\end{abstract}

1. Preliminaries. Let $H$ be a complex Hilbert space. An operator $T$ means a bounded linear transformation on $H$. For an operator $T, \sigma(T)$ denotes its spectrum and $\rho(T)$ denotes its resolvent set. An invariant subspace $Y$ of $T$ is called a spectral maximal space of $T$ if $Z \subset Y$ for any invariant subspace $Z$ of $T$ such that $\sigma(T \mid Z) \subset \sigma(T \mid Y) . \operatorname{SM}(T)$ denotes the family of all spectral maximal spaces of $T$. An operator $T$ is called weak decomposable if for any open covering $\left\{G_{1}, \ldots, G_{n}\right\}$ of $\sigma(T)$ there exists a system $\left\{Y_{1}, \ldots, Y_{n}\right\}$ in $\operatorname{SM}(T)$ such that (1) $H=\overline{Y_{1}+\cdots+Y_{n}}$ and (2) $\sigma\left(T \mid Y_{1}\right) \subset G_{i}$ for every $i=1, \ldots, n$.

An operator $T$ is said to have the property (A) or the single valued extension property if there exists no nonzero analytic function $f(z)$ such that $(z-T) f(z) \equiv 0$. If an operator $T$ has (A), then for any $x \in H$ there exists a maximal analytic extension $f_{x}(z)$ of $(z-T)^{-1} x$ such that $(z-T) f_{x}(z) \equiv x . \rho_{T}(x)$ denotes the domain of $f_{x}(z)$ and $\sigma_{T}(x)=\mathbf{C} \backslash \rho_{T}(x)$. Let $H_{T}(E)=\left\{x \in H: \sigma_{T}(x) \subset E\right\}$ for any subset $E \subset \mathbf{C}$. An operator $T$ with (A) is said to satisfy the condition (C) if $H_{T}(F)$ is closed for all closed subsets $F \subset \mathbf{C}$.

An operator $T$ is called reductive if every invariant subspace of $T$ reduces $T$.

\section{Main results.}

Lemma 1. For an operator $T$ with (A) and for a subset $E \subset \mathrm{C}$, the following assertions are equivalent.

(1) $\sigma\left(T \mid \overline{H_{T}(E)}\right) \subset E$.

(2) $H_{T}(E)$ is closed.

And in this case $H_{T}(E) \in \operatorname{SM}(T)$.

Proof. We show the implication $(1) \rightarrow(2)$. Let $x \in \overline{H_{T}(E)}$ be given, then $f(z)=$ $\left(z-T \mid \overline{H_{T}(E)}\right)^{-1} x$ is analytic and $(z-T) f(z) \equiv x$ on $\rho\left(T \mid \overline{H_{T}(E)}\right) \supset \mathbf{C} \backslash E$. Hence $\sigma_{T}(x) \subset E$ and $x \in H_{T}(E)$. Thus $H_{T}(E)$ is closed. The implication (2) $\rightarrow(1)$ and $H_{T}(E) \in \operatorname{SM}(T)$ is well known by [1, Proposition 1.3.8].

Received by the editors July 1, 1981 and, in revised form. April 2, 1982.

1980 Mathematics Subject Classification. Primary 47B20, 47B40.

Key words and phrases. Spectral operator. decomposable operator, weak decomposable operator, reductive operator. 
LEMMA 2. If an operator $T$ is reductive and weak decomposable, then $T$ satisfies (C).

Proof. Since every weak decomposable operator $T$ has (A) by [2, Theorem 2.3], we have only to show $\sigma\left(T \mid \overline{H_{T}(F)}\right) \subset F$ for all closed subsets $F \subset \mathrm{C}$ by Lemma 1 . Let $\lambda_{0} \notin F$ be given, then there exist an $\varepsilon>0$ and $G_{1}=\left\{\lambda:\left|\lambda-\lambda_{0}\right|<2 \varepsilon\right\}$ such that $G_{1} \cap F=\phi$. Let $G_{2}=\left\{\lambda:\left|\lambda-\lambda_{0}\right|>\varepsilon\right\}$, then $\left\{G_{1}, G_{2}\right\}$ is an open covering of $\sigma(T)$, hence there exist $Y_{1}, Y_{2} \in \operatorname{SM}(T)$ such that $H=\overline{Y_{1}+Y_{2}}$ and $\sigma\left(T \mid Y_{i}\right) \subset G_{i}$ for $i=1,2$. Let $P$ denote the orthogonal projection of $H$ onto $\overline{H_{T}(F)}$. Then $T P=P T$ by assumption and $\overline{H_{T}(F)}=P H=P\left(\overline{Y_{1}+Y_{2}}\right) \subset \overline{P Y_{1}+P Y_{2}}$. We show $P Y_{1}=\{0\}$. Since $Y_{1} \in \operatorname{SM}(T)$ is hyperinvariant under $T$ by [1, Proposition 1.3.2], we have $P Y_{1} \subset Y_{1}$ and $P Y_{1} \subset Y_{1} \cap \overline{H_{T}(F)}$. Let $P_{1}$ denote the orthogonal projection of $H$ onto $Y_{1}$. Then $T P_{1}=P_{1} T$, hence for any $x \in H_{T}(F)$ we have $\sigma_{T}\left(P_{1} x\right) \subset \sigma_{T}(x)$ $\cap \sigma\left(T \mid Y_{1}\right) \subset F \cap G_{1}=\phi$. Hence $P_{1} x=0$ and it follows immediately that $Y_{1} \perp$ $H_{T}(F)$. Thus $P Y_{1} \subset Y_{1} \cap \overline{H_{T}(F)}=\{0\}$ and $P Y_{1}=\{0\}$. Hence $\overline{H_{T}(F)}$ $\subset \overline{P Y_{1}+P Y_{2} \subset P Y_{2}} \subset Y_{2}$ because $P Y_{2} \subset Y_{2}$. Since $\overline{H_{T}(F)}$ is a reducing subspace of $T$, we have

$$
\sigma_{T \mid \overline{H_{T}(F)}}(x)=\sigma_{T}(x) \subset \sigma\left(T \mid Y_{2}\right) \subset G_{2}
$$

for all $x \in \overline{H_{T}(F)}$. Hence

$$
\sigma\left(T \mid \overline{H_{T}(F)}\right)=\bigcup\left\{\sigma_{T / \overline{H_{T}(F)}}(x): x \in \overline{H_{T}(F)}\right\} \subset G_{2},
$$

and thus $\lambda_{0} \neq \sigma\left(T \mid \overline{H_{T}(F)}\right)$.

THEOREM. If an operator $T$ is reductive and weak decomposable, then $T=N+Q$ where $N$ is a normal operator and $Q$ is a quasinilpotent operator commuting with $N$, i.e. $T$ is a spectral operator with a normal scalar part.

Proof. We show first $\sigma\left(T \mid H_{T}(F)^{\perp}\right) \subset \overline{\mathbf{C} \backslash F}$ for all closed subsets $F \subset \mathbf{C}$. Let $\lambda_{0} \notin \overline{\mathbf{C} \backslash F}$ be given, then there exists an $\varepsilon>0$ and $G_{1}=\left\{\lambda:\left|\lambda-\lambda_{0}\right|<2 \varepsilon\right\}$ such that $G_{1} \cap \overline{\mathbf{C} \backslash F}=\varnothing$. Let $G_{2}=\left\{\lambda:\left|\lambda-\lambda_{0}\right|>\varepsilon\right\}$, then $\left\{G_{1}, G_{2}\right\}$ is an open covering of $\sigma(T)$, hence there exist $Y_{1}, Y_{2} \in \operatorname{SM}(T)$ such that $H=\overline{Y_{1}+Y_{2}}$ and $\sigma\left(T \mid Y_{i}\right)$ $\subset G_{i}$ for $i=1,2$. Let $P$ denote the orthogonal projection of $H$ onto $H_{T}(F)^{\perp}$. Then $T P=P T$ by assumption and $H_{T}(F)^{\perp}=P H=P\left(\overline{Y_{1}+Y_{2}}\right) \subset \overline{P Y_{1}+P Y_{2}}$. We show $P Y_{1}=\{0\}$. Let $x \in Y_{1}$ be given, then $\sigma_{T}(P x) \subset \sigma_{T}(x) \subset \sigma\left(T \mid Y_{1}\right) \subset G_{1} \subset F$. Hence $P x \in H_{T}(F) \cap H_{T}(F)^{\perp}=\{0\}$ and $P Y_{1}=\{0\}$. Hence $H_{T}(F)^{\perp} \subset \overline{P Y_{1}+P Y_{2}}$ $\subset \overline{P Y}_{2} \subset Y_{2}$. Since $H_{T}(F)^{\perp}$ is a reducing subspace of $T$, we have $\sigma\left(T \mid H_{T}(F)^{\perp}\right) \subset$ $\sigma\left(T \mid Y_{2}\right) \subset G_{2}$, and thus $\lambda_{0} \notin \sigma\left(T \mid H_{T}(F)^{\perp}\right)$. Hence $\sigma\left(T \mid H_{T}(F)^{\perp}\right) \subset \overline{\mathbf{C} \backslash F}$ and $H_{T}(F)^{\perp} \subset H_{T}(\overline{\mathbf{C} \backslash F})$. Hence $H=H_{T}(F)+H_{T}(F)^{\perp} \subset H_{T}(F)+H_{T}(\overline{\mathbf{C} \backslash F})$ and so $H=H_{T}(F)+H_{T}(\overline{\mathbf{C} \backslash F})$ for all closed subsets $F \subset \mathbf{C}$. Wadhwa [5, Corollary 4] shows that if a reductive operator $T$ has (A) and satisfies (C) and $H=H_{T}(F)+$ $H_{T}(\overline{\mathbf{C} \backslash F})$ for all closed subsets $F \subset \mathbf{C}$, then $T$ is a spectral operator with a normal scalar part. Hence the proof is completed by Lemma 2 .

REMARK. We can relax the condition of weak decomposability by weak 2 -decomposability.

ACKnOwledgements. The author wishes to thank the late Professor T. Saito and Professor T. Yoshino for helpful comments. And the author wishes to thank the referee for his exact reading of this paper. 


\section{REFERENCES}

1. I. Colojoară and C. Foias,, Theory of generalized spectral operators, Gordon \& Breach. New York, 1968

2. A. A. Jafarian, Weak and quasi-docomposable operators, Rev. Roumaine Math. Pures Appl. 22 (1977), 195-212.

3. __ On reductive operators, Indiana Univ. Math. J. 23 (1974), 607-613.

4. B. L. Wadhwa, Decomposable and spectral operators on a Hilbert space, Proc. Amer. Math. Soc. 40 (1973), 112-114.

5. A note on reductive operators, Acta Sci. Math. (Szeged) 38 (1976), 187- 189.

Tohoku College of Pharmacy, Komatsushima, Sendai, 983, Japan 